Oscar Hernán Jalil Guiteras

Metodologia de Análise Global para o Desenvolvimento de um Campo de Gás Natural

Dissertação de Mestrado

Dissertação apresentada como requisito parcial para obtenção do título de Mestre pelo Programa de PósGraduação em Engenharia Mecânica do Departamento de Engenharia Mecânica do Centro Técnico Científico da PUC-Rio.

Orientador: Luís Fernando A. Azevedo Co-orientador: Oswaldo Antune Pedrosa Jr.

Rio de Janeiro

Agosto de 2003 

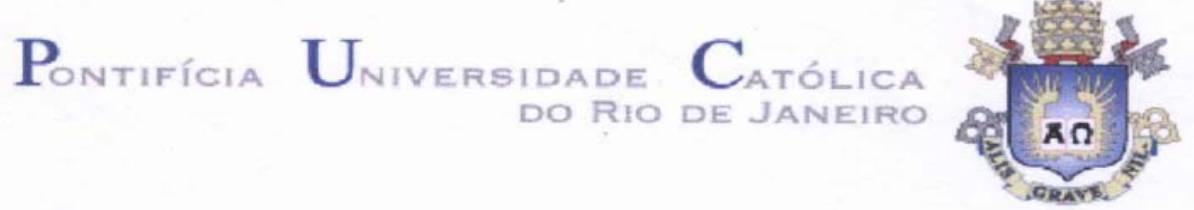

Oscar Hernán Jalil Guiteras

\section{Metodologia de Análise Global para o \\ Desenvolvimento de um Campo de \\ Gás Natural}

Dissertação apresentada como requisito parcial para obtenção do título de Mestre pelo Programa de PósGraduação em Engenharia Mecânica da PUC-Rio. Aprovada pela Comissão Examinadora abaixo assinada.

\section{Prof. Luís Fernando Alzuguir Azevedo}

Orientador

PUC-Rio - Pontifícia Universidade Católica do Rio de Janeiro

Prof. Oswaldo Antune Pedrosa Jr. Co-Orientador PUC-Rio - Pontifícia Universidade Católica do Rio de Janeiro

Prof. Arthur Martins Barbosa Braga PUC-Rio - Pontifícia Universidade Católica do Rio de Janeiro

Prof. Marcos Sebastião de Paula Gomes PUC-Rio - Pontifícia Universidade Católica do Rio de Janeiro

Prof. Ney Augusto Dumont Coordenador(a) Setorial do Centro Técnico Científico - PUC-Rio 
Todos os direitos reservados. É proibida a reprodução total ou parcial do trabalho sem autorização da universidade, do autor e do orientador.

\section{Oscar Hernán Jalil Guiteras}

Graduou-se em Engenharia em Petróleo na Universidade Autônoma Gabriel Rene Moreno (UAGRM), em Santa Cruz de la Sierra - Bolívia, em julho de 1997, nesse mesmo ano realizou estágio na Empresa Petroleira CHACO S.A., adquirindo grandes conhecimentos Técnico - Científicos. Em 1998-2001 trabalhou na Empresa MAXUS BOLIVIA Inc. como Engenheiro em Produção, desenvolvendo diversos projetos na área de atuação. No período 2001 2003 cursou o Mestrado em Engenharia Mecânica na Pontifícia Universidade Católica do Rio de Janeiro - PUCRio

Ficha Catalográfica

Jalil Guiteras Oscar Hernán

Metodologia de análise global para o desenvolvimento de um campo de gás natural / Oscar Hernán Jalil Guiteras; orientador: Luís Fernando A. Azevedo; co-orientador: Oswaldo Antune Pedrosa Jr. - Rio de Janeiro : PUC, Departamento de Engenharia Mecânica, 2003.

285 f. : il. ; $30 \mathrm{~cm}$

Dissertação (mestrado) - Pontifícia Universidade Católica do Rio de Janeiro, Departamento de Engenharia Mecânica.

Inclui referências bibliográficas.

1. Engenharia Mecânica - Teses. 2. Produção de gás natural. 3. Fluxo de gás natural em tubulações horizontal e vertical. 4. Relação de comportamento do fluxo de entrada. I. Azevedo, Luís Fernando A. II. Pedrosa Jr., Oswaldo Antune. III. Pontifícia Universidade Católica do Rio de Janeiro. Departamento de Engenharia Mecânica. III. Título. 


\section{Agradecimentos}

A Deus pela fé, força e perseverança na trajetória de minha vida.

À família Bravo Jalil pelo carinho, apoio e confiança.

Aos professores Luís Fernando A. Azevedo e Oswaldo A. Pedrosa Jr. pelo apoio, orientação e amizade no desenvolvimento deste trabalho.

Ao Departamento de Engenharia Mecânica da PUC - RIO, pelo apoio dispensado por todos os professores e funcionários. 


\section{Resumo}

Jalil Guiteras Oscar Hernán. Metodologia de análise Global para o desenvolvimento de um campo de gás natural. Rio de Janeiro, 2003. 285p. Dissertação de Mestrado - Departamento de Engenharia Mecânica, Pontifícia Universidade Católica do Rio de Janeiro.

Para que ocorra o fluxo de fluidos em um sistema de produção é necessário que a energia dos fluidos no reservatório seja capaz de superar as perdas de carga nos diversos componentes do sistema. Os fluidos têm que escoar do reservatório aos separadores na superfície, passando pelas tubulações de produção dos poços, pelos equipamentos de cabeça de poço e pelas linhas de surgência. O projeto de um sistema de produção não deve ser executado considerando independentemente o desempenho do reservatório e o cálculo do fluxo nas tubulações de produção e nas linhas e equipamentos de superfície. A avaliação do desempenho de um sistema de produção de gás requer a aplicação de um método de análise total que considere simultaneamente o escoamento nos diversos segmentos do sistema. A análise total de um sistema de produção, pode ser efetuada por um método gráfico - analítico a ser empregado no desenho de completação de poços de gás e petróleo com ou sem levantamento artificial. Em termos de conceito de análise total, um sistema de produção é constituído basicamente pelos elementos: reservatório, tubo de produção vertical, linhas de fluxo horizontal e separador, incluindo válvulas de fundo de poço e choke superficiais, onde ocorrem uma certa perda de pressão relacionada com a vazão. O comportamento de fluxo de cada elemento do sistema total de produção é representado por uma equação que relaciona a pressão num nó selecionado e a vazão de produção. O cálculo seqüencial das pressões nos diferentes nós dos diversos elementos do sistema permite que a vazão de produção do poço seja determinada. Para calcular a relação da vazão com as mudanças de pressão que ocorrem durante o transporte do fluido à superfície e conseqüente variação das propriedades físicas do fluido, efetuou-se uma revisão dos conceitos de engenharia de reservatório, correlações de fluxo em tubulações verticais, horizontais e restrições. Finalmente, realizou-se uma análise de sensibilidade da metodologia ao emprego das diferentes relações de performance de fluxo e dos métodos de cálculo de fluxo em poços e linhas.

\section{Palavras-chave}

Produção de Gás Natural; Fluxo de Gás Natural em tubulações horizontal e vertical; Relação de Comportamento do Fluxo de Entrada. 


\section{Abstract}

Jalil Guiteras Oscar Hernán. Total Analysis Methodology For The Development Of A Natural Gas Field. Rio de Janeiro, 2003. 285p. Master's Degree Dissertation - Mechanical Engineering Department, Pontifical Catholic University of Rio de Janeiro.

For the flow of fluids to occur in a production system, the fluids' energy in the reservoir must be capable of overcoming load losses along various system components. Fluids must flow from the reservoir to the surface separators through the wells' tubing, wellhead equipment and flowing lines. The production system design must not be executed by considering separately the performance of the reservoir and the flow calculation across the tubing, surface equipment and lines. The evaluation of a gas production system performance requires applying a total analysis method that considers the drainage at various system segments simultaneously. The total analysis of a production system can be effected through a graphical-analytical method to be used for the completion design of oil and gas wells with or without artificial lift. In terms of total analysis concept, a production system is basically comprised of the following components: reservoir, vertical tubing, horizontal flow lines and separator, including bottom-hole valves and surface choke, where some pressure loss occurs in relation to the flow rate. The flow behavior of each component in a total production system is represented by an equation that relates the pressure at one selected node and the production flow rate. The sequential pressure calculation at the different nodes of various system components allows determining the well's production flow rate. In order to calculate the relationship between the flow rate and the pressure changes that occur during fluid transportation to the surface, with the resulting variation of the fluid's physical properties, we reviewed the concepts of reservoir engineering, flow correlations in vertical and horizontal tubing, and restrictions. Finally, we proceeded to analyze the methodology's sensitivity to the use of different flow performance relations and flow calculation methods in wells and lines.

\section{Key Words}

Natural Gas Production; Natural Gas Flow in Pipelines and Tubing; Inflow Performance Relationship for Gas Wells. 


\section{Sumário}

1 Introdução 25

1.1 Introdução 25

1.1.1 Historia e previsão do consumo do gás natural no Mundo 25

1.1.2 História Do gás natural no Brasil 26

1.1.3 Evolução do gás por setores 27

1.1.4 Reservas provadas de gás natural da Pretobras por região 28

1.1.5 Expectativas para o mercado de gás do Brasil 28

1.1.6 Reservas de gás natural na América do Sul 29

1.1.7 Mercado do gás natural e perspectivas na América do Sul 29

1.1.8 Demanda de gás natural na América do Sul 30

1.1.9 Reservas de gás natural na Bolívia 31

1.1.10 Reservas e balanço de gás natural na Bolívia 31

1.1.11 Contratos de risco compartilhado na Bolívia 32

1.1.12 Mercado potencial para o gás naturalda Bolívia 32

1.1.13 Gasoduto Bolívia - Brasil 33

1.2 Objetivos 36

$\begin{array}{lll}1.3 & \text { Conteúdo do trabalho desenvolvido } & 37\end{array}$

2 Comportamento Termodinâmico de Fluidos no Reservatório 39

2.1 Introdução 39

2.2 Diagrama de fases pressão - temperatura 39

2.2.1 Fase envolvente $\quad 40$

$\begin{array}{lll}2.2 .2 & \text { Linhas de qualidade } & 40\end{array}$

$\begin{array}{lll}2.2 .3 & \text { Ponto crítico } & 40\end{array}$

2.2.4 Ponto cricondentérmico 40

2.2.5 Ponto cricondenbárico 40

2.2.6 Curva ponto de bolha 41

2.2.7 Curva ponto de orvalho 41 
2.3 Classificação dos reservatórios

2.4 Reservatórios de petróleo 41

2.4.1 Reservatório de petróleo subsaturado 41

2.4.2 Reservatório de petróleo saturado 41

2.4.3 Reservatório de capa de gás 42

2.5 Reservatórios de gás 48

2.5.1 Reservatório de condensação retrógrada de gás 48

2.5.2 Reservatório de condensado perto no ponto crítico 49

2.5.3 Reservatório de gás úmido 50

2.5.4 Reservatório de gás seco 51

3 Análise Reservatório - Poço 53

3.1 Introdução 53

3.1.1 Lei de Darcy 55

3.1.1.1 Fluxo linear $\quad 55$

3.1.1.2 Fluxo radial 58

3.2 Regimes de fluxo 61

3.2.1 Fluxo em estado estável $\quad 61$

3.2.2 Fluxo em estado transiente 65

3.2.3 Fluxo em estado pseudo estável $\quad 71$

3.3 Dano à formação próxima ao poço 75

3.4 Efeito de turbulência 77

3.5 Relação do comportamento do fluxo de entrada em poços de gás $\quad 79$

$\begin{array}{lll}\text { 3.5.1 Tratamento simplificado } & 79\end{array}$

3.5.1.1 Teste seqüencial 79

3.5.1.2 Teste de fluxo isócrono 83

3.5.1.3 Teste de fluxo isócrono modificado 84

3.5.2 Laminar inercial turbulento 87

3.5.2.1 Forma quadrática - pressão ao quadrado 87

3.5.2.2 Forma quadrática - pressão 90

3.5.2.3 Forma potencial real do gás (pseudopressões) 91 
4 Análise da Coluna de Produção e Linha de Fluxo 92

4.1 Introdução 92

$\begin{array}{lll}4.2 & \text { Equação da energia } & 93\end{array}$

4.3 Número de Reynolds $\quad 97$

4.4 Rugosidade relativa 98

4.5 Determinação do fator de atrito 100

4.5.1 Fluxo laminar de fase simples 100

4.5.2 Fluxo turbulento de fase simples 102

4.5.2.1 Tubulações lisas 102

4.5.2.2 Tubulações rugosas 102

4.6 Fluxo de fase simples 103

$\begin{array}{lll}4.7 & \text { Fluxo nos poços de gás } & 104\end{array}$

4.7.1 Pressão estática de fundo do poço 105

4.7.1.1 Método da temperatura e compressibilidade média 105

4.7.1.2 Método cullender e smith 107

4.7.2 Pressão dinâmica de fundo do poço 109

4.7.2.1 Método da temperatura e compressibilidade média 110

4.7.2.2 Método cullender e smith 111

$\begin{array}{lll}4.8 & \text { Fluxo de gás em linhas de fluxo } & 115\end{array}$

4.9 Velocidade de erosão 118

5 Análise do Fluxo de Gás Através de Chokes 120

$\begin{array}{ll}5.1 \text { Introdução } & 120\end{array}$

5.2 Chokes superficiais 122

5.2.1 Tipo positivo 122

5.2.1 Tipo ajustáveis 122

5.3 Modelos de fluxo 122

5.3.1 Fluxo subcrítico 122

$\begin{array}{lll}\text { 5.3.2 Fluxo crítico } & 124\end{array}$

$\begin{array}{lll}5.4 & \text { Fluxo de gás através de restrições } & 125\end{array}$ 
6 Análise do Sistema de Produção 127

6.1 Introdução 127

6.2 Revisão bibliográfica 130

6.3 aspectos gerais da análise global de um sistema de produção 133

6.3.1 Pressões constantes 134

6.3.2 Análise do sistema no fundo do poço 136

6.3.2.1 Seleção do tamanho do tubo de produção 137

6.3.2.2 Efeito de depleção 139

6.3.3 Análise do sistema na cabeça do poço 139

6.3.4 Análise do sistema no separador 140

6.3.5 Análise do sistema na restrição superficial 147

7 Análise de Aplicação 151

7.1 Introdução 151

7.2 Dados básicos 151

7.3 Determinação das propriedades do gás natural poço PUC - X1 153

7.3.1 Peso molecular aparente 154

7.3.2 Densidade 154

$\begin{array}{lll}\text { 7.3.3 Fator compressibilidade } Z & 155\end{array}$

7.3.3.1 Correlação de Brill \& Beggs 155

7.3.3.2 Correlação de Hall - Yarborough 155

7.3.4 Massa específica 156

7.3.5 Fator volume de formação 156

7.3.6 Compressibilidade isotérmica 156

7.3.6.1 Propriedades pseudo críticas 156

7.3.6.2 Correções das propriedades pseudo críticas 157

7.3.6.3 Propriedades pseudo reduzidas 157

7.3.7 viscosidade do gás natural 158

7.3.7.1 Método de Carr, Kobayashi e Burrows 158

7.3.7.2 Método de Lee, Gonzales e Eakin 159 
7.4 Análise reservatório - poço

7.4.1 Vazão de fluxo mínimo para o levantamento de líquido contínuo e vazão de erosão

160

7.4.2 Método simplificado

162

7.4.3 Método de pressão 166

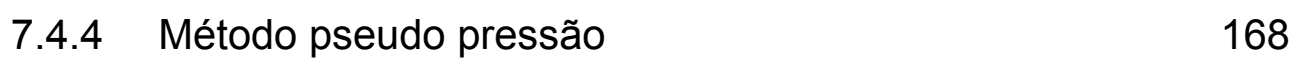

7.4.5 Cálculo do AOF através de dados de reservatório 179

7.5 Análise na coluna de produção e linha de fluxo 182

7.5.1 Coluna de produção 182

7.5.1.1 Método da temperatura e compressibilidade média 182

7.5.1.2 Método cullender e smith 183

$\begin{array}{ll}\text { 7.5.2 Linha de fluxo } & 187\end{array}$

7.5.2.1 Método da temperatura e compressibilidade média

7.5.2.2 Weymouth 188

7.5.2.3 Panhandle A e B 190

7.6 Análise do tamanho do choke superficial 200

7.7 Análise do sistema na entrada ao do separador 205

7.8 Análise total do sistema 207

8 Conclusões e Recomendações 210

9 Referências bibliográficas 213

Apêndice A Propriedades do gás natural 215

Apêndice B Pressão de orvalho para gás natural 244

Apêndice C Determinação da Função Pseudo Pressão 245

Apêndice D Interpretação Teste de Formação DST, Poço PUC - X1 251

Apêndice E Análise Instrumental Cromatografia Gasosa 254

Apêndice F Sistema de Automatização do Sistema Global para um Poço Gasífero 261

Apêndice G Sistemas e Conversões de Unidades 282 


\section{Lista de figuras}

Figura 1.1 História e previsão do consumo do gás natural no mundo 26

Figura 1.2 Evolução do gás natural por setores no Brasil 27

Figura 1.3 Gasoduto Bolívia - Brasil 34

Figura 2.1 Diagrama de fases pressão - temperatura 39

Figura 2.2 Típico diagrama P-T para petróleo negro 43

Figura 2.3 Curva de encolhimento líquido para petróleo negro 43

Figura 2.4 Típico diagrama P-T para petróleo de baixo encolhimento 45

Figura 2.5 Curva para baixo encolhimento de petróleo 45

Figura 2.6 Típico diagrama P-T para petróleo volátil 46

Figura 2.7 Curva de encolhimento de líquido para petróleo volátil $\quad 46$

Figura 2.8 Diagrama de fase esquemático para petróleo próximo ao ponto crítico $\quad 47$

Figura 2.9 Curva típica de encolhimento de líquido para petróleo próximo ao ponto crítico $\quad 47$

Figura 2.10 Diagrama de fase mostrando as regiões de condensação retrógrada 49

Figura 2.11 Típico diagrama de fase para um reservatório de gás condensado próximo ao ponto crítico 50

Figura 2.12 Diagrama de fase para gás úmido 51

Figura 2.13 Diagrama de fase para gás seco 52

Figura 3.1 Curvas IPR típicas 54

Figura 3.2 Geometria para fluxo laminar 57

Figura 3.3 Fluxo radial 59

Figura 3.4 Distribuição radial de pressão em regime estável 62

Figura 3.5 Distribuição radial de pressão em regime pseudo estável 73

Figura 3.6 Queda de pressão adicional devido a dano à formação 75

Figura 3.7 Teste de fluxo convencional 82

Figura 3.8 Esquema para teste de fluxo convencional 82

Figura 3.9 Teste de fluxo isócrono 85 
Figura 3.10 Esquema para teste de fluxo isócrono

Figura 3.11 Curva de comportamento de fluxo de entrada 86

Figura 3.12 Teste de fluxo isócrono modificado 87

Figura 3.13 Análise gráfica para determinar A e B 89

Figura 4.1 Direções de fluxo na tubulação 92

Figura 4.2 Fluxo de fluido em tubulação inclinada 95

Figura 4.3 Equilíbrio de forças 96

Figura 4.4 Fatores de atrito para qualquer tipo de tubulação comercial 99

Figura 4.5 Valores da rugosidade relativa e fator de atrito 101

Figura 5.1 Esquema do choke 120

Figura 5.2 Localização do choke 121

Figura 5.3 Porta orifícios tipo positivo 123

Figura 5.4 Chokes positivos 123

Figura 5.5 Válvula de agulha regulável 124

Figura 6.1 Sistema simples de produção 127

Figura 6.2 Possíveis perdas da pressão em um sistema completo 128

Figura 6.3 Determinação da capacidade de fluxo 135

Figura 6.4 Colocação de vários nós 136

Figura 6.5 Componentes do reservatório 138

Figura 6.6 Condições instáveis por efeito do diâmetro 141

Figura 6.7 Poço restringido pelo sistema de tubulação 141

Figura 6.8 Efeito do tamanho da tubulação 142

Figura 6.9 Efeito da mudança de diâmetro da tubulação sobre a capacidade do poço 142

Figura 6.10 Previsão das vazões futuras 143

Figura 6.11 Efeito de depleção 143

Figura 6.12 Componentes de separador e linha de fluxo 144

Figura 6.13 Componentes do reservatório e tubulação 144

Figura 6.14 Solução em cabeça do poço 145

Figura 6.15 Nó solução no separador 146

Figura 6.16 Efeitos da pressão no separador 146

Figura 6.17 Nó solução no choke de superfície 148

Figura 6.18 Avaliação do choke de superfície 149 
Figura 6.19 Comportamento total do sistema incluindo choke de superfície

Figura 6.20 Comportamento do choke

Figura 6.21 Comportamento do sistema para diversos diâmetros de choke 


\section{Lista de tabelas}

Tabela 1.1 Reservas provadas de gás natural da Petrobrás 28

Tabela 1.2 Reservas provadas de gás natural na América do Sul 29

Tabela 1.3 Evolução das reservas de gás natural certificadas da Bolívia

Tabela 1.4 Relação de reservas / consumo para os próximos 70 anos

Tabela 1.5 Contratos de risco compartilhado na Bolívia.

Tabela 1.6 Volumes de contrato compra - venda de gás natural, Bolívia - Brasil

Tabela 1.7 Valores e preços do gás natural, Bolívia - Brasil 35

Tabela 3.1 Unidades da lei de Darcy 56

Tabela 4.1 Regime de fluxo relacionado com o número de Reynolds

Tabela 4.2 Valores de rugosidade absoluta

Tabela 4.3 Valores de C para diversas unidades 117

Tabela 4.4 Valores das constantes ai 118

Tabela 7.1 Parâmetros do reservatório 152

Tabela 7.2 Parâmetros do fluido 152

Tabela 7.3 Dados do teste seqüencial 152

Tabela 7.4 Composição do gás natural, poço PUC - X1 153

Tabela 7.5 Peso molecular aparente 154

Tabela 7.6 Fator Z - Brill \& Beggs 155

Tabela 7.7 Fator $Z$ - Hall-Yarborough 155

Tabela 7.8 Resumo dos valores obtidos de $Z$

Tabela 7.9 Massa específica do gás natural - Poço PUC - X1 156

Tabela 7.10 Fator volume de formação 156

Tabela 7.11 Propriedades pseudo críticas da Mistura 157

Tabela 7.12 Viscosidade do gás natural 160

Tabela 7.13 Vazão de fluxo mínima para o levantamento de líquido contínuo 161

Tabela 7.14 Velocidade e Vazão de Erosão 161 
Tabela 7.15 Análise Simplificado

Tabela 7.16 Curva do comportamento do reservatório (CCR)

Tabela 7.17 Análise da forma quadrática - pressão

Tabela 7.18 Curva do comportamento do reservatório (CCR)

Tabela 7.19 Cálculo da função pseudo - pressão $\mathrm{m}(\mathrm{p})$

Tabela 7.20 Análise pseudo - pressão

Tabela 7.21 Curva do comportamento do reservatório (CCR) 175

Tabela 7.22 Resumo dos valores de AOF (teste)

Tabela 7.23 Curvas do comportamento do reservatório (CCR), dados teste

Tabela 7.24 Curva do comportamento do reservatório (CCR), dados do reservatório

Tabela 7.25 Valores estimados da pressão na cabeça do poço para diferentes Diâmetros (método temperatura e compressibilidade Média)

Tabela 7.26 valores estimados da pressão na cabeça do poço para diferentes diâmetros (método Cullender e Smith)

Tabela 7.27 Valores estimados da pressão na cabeça do poço para diferentes diâmetros (método temperatura e compressibilidade Média)

Tabela 7.28 Valores estimados da pressão na cabeça do poço para diferentes diâmetros (método Weymouth)

Tabela 7.29 Valores Estimados da Pressão na Cabeça do Poço para Diferentes Diâmetros (método Panhandle A)

Tabela 7.30 Valores estimados da pressão na cabeça do poço para diferentes diâmetros (método Panhandle B)

Tabela 7.31 Capacidade de fluxo para diferentes diâmetros do tubo de produção ( $P_{\mathrm{wf}}$, método pseudo pressão)

Tabela 7.32 Capacidade de fluxo para diferentes diâmetros do tubo de produção ( $P_{\mathrm{wf}}$, método simplificado)

Tabela 7.33 Capacidade de fluxo para diferentes diâmetros do tubo de produção ( $P_{\text {wf }}$ método pressão) 
Tabela 7.35 Valores de vazão e pressão para diferentes diâmetros de choke

Tabela 7.36 Análise do sistema no separador 205

Tabela 7.37 Análise total do sistema 


\section{Nomenclatura}

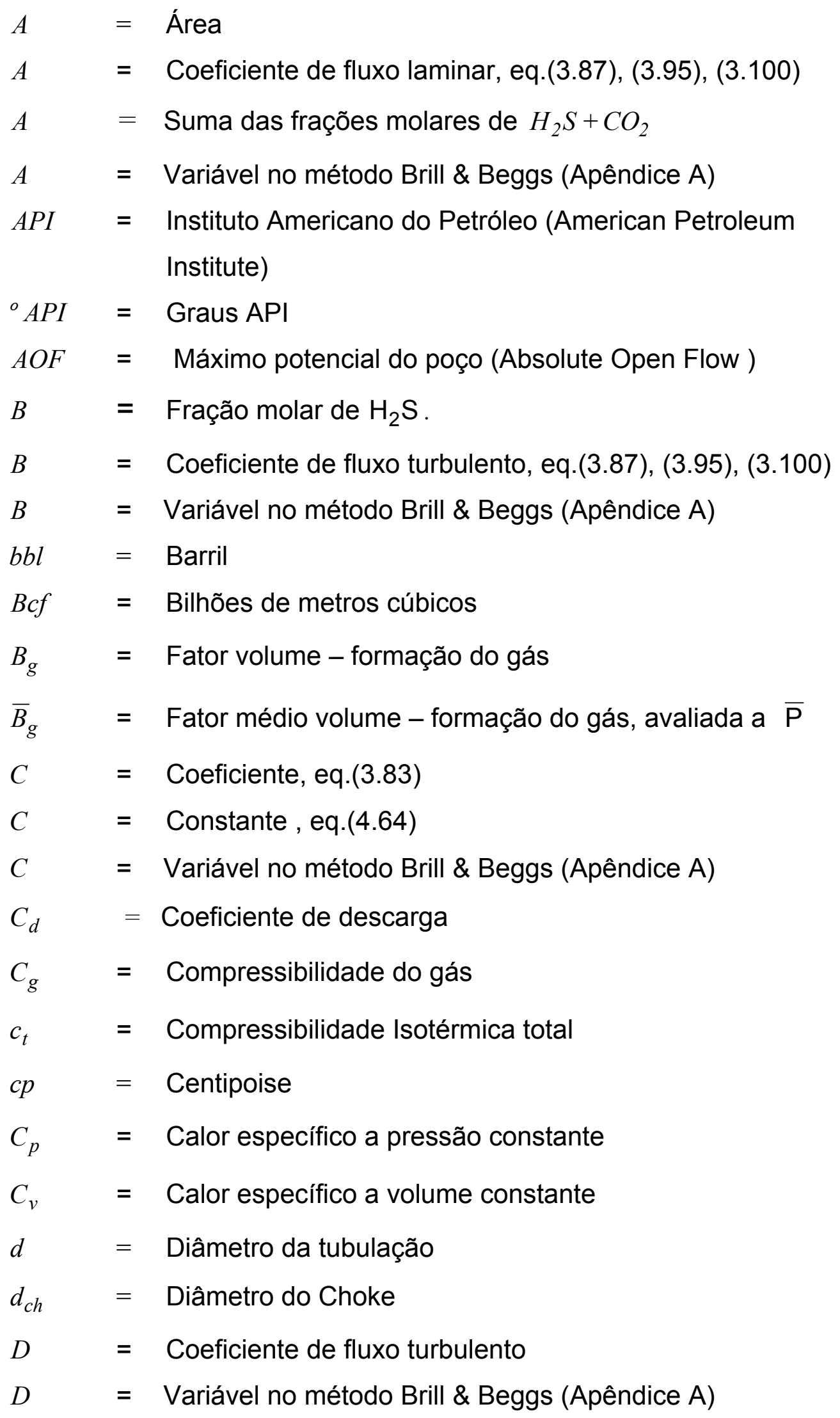




$$
\begin{aligned}
& D S T=\text { Drillsteam Test } \\
& E \quad=\quad \text { Fator de eficiência } \\
& e \quad=\text { Rugosidade absoluta } \\
& F \quad=\quad \text { Coeficiente de fluxo não Darcy } \\
& g=\text { Aceleração gravitacional } \\
& g_{c}=\text { Constante gravitacional } 32.17 l b_{m} f t / l b_{f} \sec ^{2} \\
& f=\text { Fator de atrito } \\
& f^{\prime}=\text { Fator de atrito Fanning } \\
& { }^{o} \mathrm{~F} \quad=\text { Grau Fahrenheit } \\
& F \quad=\quad \text { Fator de atrito de Nikuradse } \\
& f t=\text { Pé (feet) } \\
& h=\text { Entalpia. } \\
& h=\text { Espessura do reservatório } \\
& H \quad=\text { Profundidade do poço } \\
& I_{w h}=\text { Integral estática avaliada a } p_{w h} \text { e } T_{w h} \\
& I_{m s}=\text { Integral estática avaliada a } p_{m s} \text { e } \bar{T} \\
& I_{w s} \quad=\quad \text { Integral estática avaliada a } p_{w s} \text { e } T_{w s} \\
& I_{t f} \quad=\quad \text { Integral de fluxo avaliada a } p_{w h} \text { e } T_{w h} \\
& I_{m f} \quad=\quad \text { Integral de fluxo avaliada a } p_{m f} \text { e } T_{m f} \\
& I_{w f} \quad=\quad \text { Integral de fluxo avaliada a } p_{w f} \text { e } T_{w f} \\
& I P R=\text { Relação de comportamento do fluxo de entrada (inflow } \\
& \text { performance relationship) } \\
& \text { in } \quad=\text { Polegada (inch) } \\
& k=\text { Permeabilidade } \\
& k_{g} \quad=\quad \text { Permeabilidade do gás } \\
& k=\text { Relação de calores específicos } \\
& k=\text { Contador de iterações } \\
& K=\quad \text { Variável no método Lee, Gonzalez e Eakin } \\
& L=\text { Comprimento } \\
& l_{w} \quad=\quad \text { Perda de trabalho, devido à irreversibilidade } \\
& \log \quad=\quad \text { Logaritmo decimal }
\end{aligned}
$$




$$
\begin{aligned}
& m \quad=\text { massa } \\
& m \quad=\quad \text { Pendente } \\
& m^{3} \quad=\quad \text { Metros cúbicos } \\
& m d=\text { Milidarcys } \\
& m(p) \quad=\text { Pseudopressão do gás real desde, } 0 \text { até } p_{R} \\
& m\left(p_{w}\right)=\text { Pseudopressão do gás real, desde } 0 \text { até } p_{w f} \\
& M \quad=\text { peso molecular } \\
& M_{a} \quad=\quad \text { Peso molecular aparente } \\
& M_{i} \quad=\quad \text { Peso molecular do componente } \mathrm{i} \\
& M D=\text { Profundidade medida (measured depth) } \\
& \text { Mscfd = Mil pés cúbicos dia (thousand standart cubic feet day) } \\
& M M \mathrm{~m}^{3} / d=\text { Milhões de metros cúbicos } \\
& n=\text { Exponente, eq.(3.83) } \\
& n=\text { Número de libras-mol } \\
& n_{c} \quad=\quad \text { Número de componentes } \\
& N_{R e} \quad=\quad \text { Número de Reynolds } \\
& \text { psia }=\text { Libras por polegada quadrada absoluta (pounds per square } \\
& \text { inch absolute) } \\
& \text { psi }=\text { Libras por polegada quadrada (pounds per square inch) } \\
& \text { ppm = Partes por milhão } \\
& p \quad=\text { Pressão } \\
& \bar{P} \quad=\quad \text { Pressão média } \\
& p_{1} \quad=\quad \text { Pressão a montante do Choke, eq.(5.1) } \\
& p_{2}=\text { Pressão a jusante do Choke, eq.(5.1) } \\
& p_{c} \quad=\text { Pressão crítica } \\
& p_{d} \quad=\text { Pressão de orvalho } \\
& p_{r} \quad=\text { Pressão reduzida, }(\text { Apêndice } \mathrm{A}) \\
& P_{p r} \quad=\text { Pressão pseudo-reduzida } \\
& P_{c i}=\text { Pressão pseudo-crítica do componente } \mathrm{i}
\end{aligned}
$$




$$
\begin{aligned}
& P_{p c} \quad=\text { Pressão pseudo-crítica } \\
& p_{s c}=\text { Pressão a condições normais, } 14,7 \text { psia } \\
& \bar{P}_{r} \quad=\quad \text { Pressão média do reservatório } \\
& P_{w f s}=\text { Pressão de fluxo nos canhoneados } \\
& P_{w f} \quad=\text { Pressão de fluxo no fundo do poço } \\
& p_{w s}=\text { Pressão estática no fundo do poço } \\
& p_{m s}=\text { Pressão estática à profundidade média da tubulação, } \mathrm{H} / 2 \\
& p_{w h}=\text { Pressão de fluxo e estática na cabeça do poço } \\
& P_{m f}=\text { Pressão de fluxo à profundidade média da tubulação, } \mathrm{H} / 2 \\
& p_{D} \quad=\quad \text { Queda de pressão, para um } t_{D} \\
& q=\text { Vazão } \\
& q_{e} \quad=\quad \text { Vazão de fluxo de erosão } \\
& q_{s c}=\text { Vazão de fluxo de gás a condições de normais } \\
& q_{g} \quad=\quad \text { Vazão de fluxo de gás } \\
& q_{g(\min )}=\text { Vazão de fluxo mínimo para o levantamento contínuo de } \\
& \text { líquidos } \\
& q_{g r} \quad=\quad \text { Vazão de fluxo do gás, para um raio } r \\
& Q=\text { Transferência de calor. } \\
& r \quad=\quad \text { Distância radial } \\
& r_{e} \quad=\text { Raio de drenagem } \\
& r_{w} \quad=\text { Raio do poço } \\
& R \quad=\quad \text { Constante universal dos gases } \\
& { }^{o} R \quad=\text { Grau Rankine } \\
& R C G=\text { Relação água - condensado } \\
& R W G=\text { Relação água - gás } \\
& s=\text { Dano à formação perto do poço } \\
& s=\text { Entropia. } \\
& s c f=\text { Pés cúbicos em condições standard (standard cubic feet) }
\end{aligned}
$$




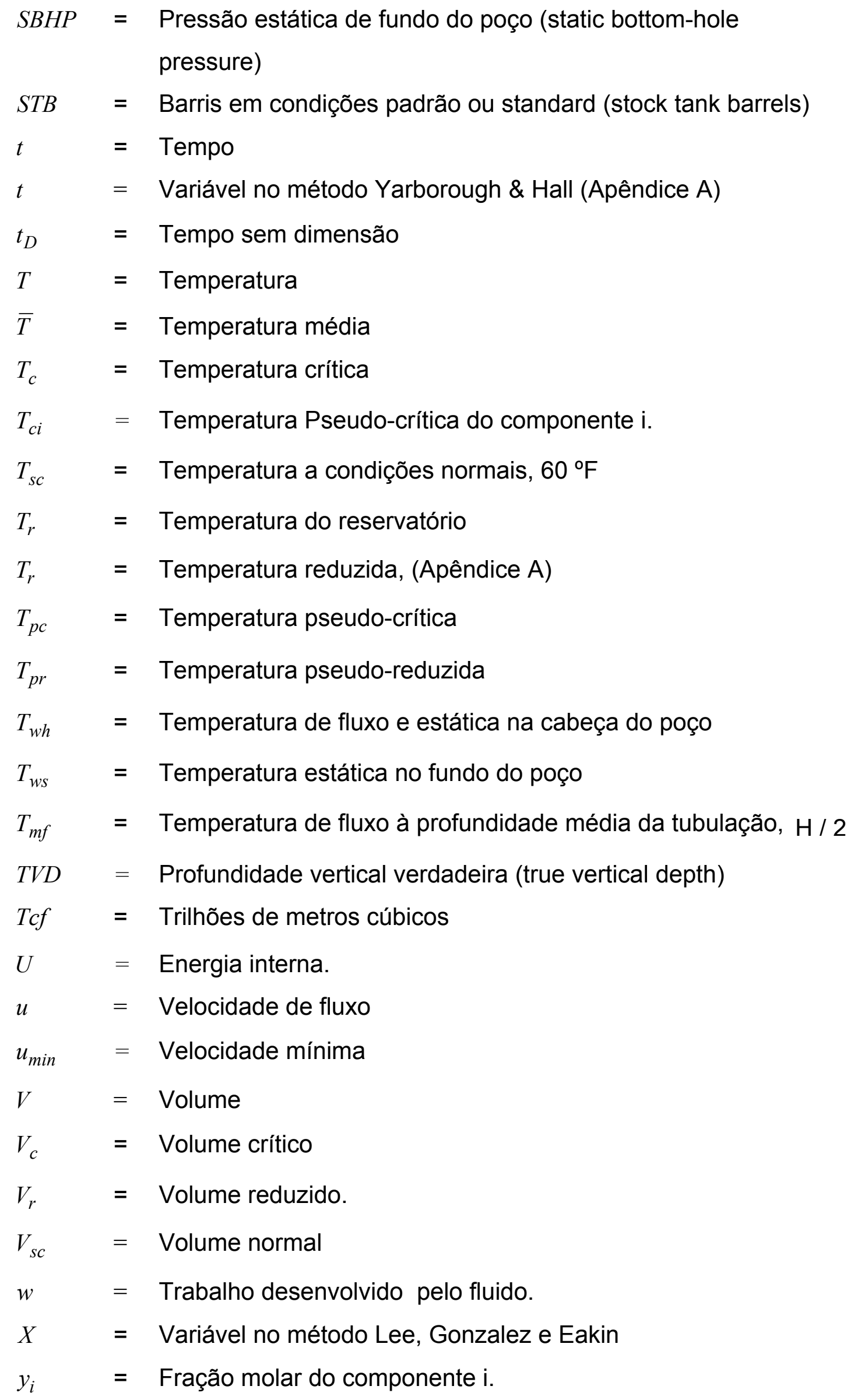


$Y=$ Variável no método Lee, Gonzalez e Eakin

$Z \quad=$ Fator de compressibilidade para p e T

$\bar{Z}=$ Fator de compressibilidade para $\overline{\mathrm{P}}, \overline{\mathrm{T}}$

$Z_{s c}=$ Fator de compressibilidade a condições normais $\cong 1$

\section{Símbolos Gregos}

$\beta=$ Coeficiente turbulento de velocidade

$\Delta \quad=$ Decremento

$\varepsilon \quad=\quad$ Fator de ajuste das propriedades pseudo críticas

$\phi \quad=$ Porosidade

$\gamma_{g} \quad=$ Densidade do gás

$\mu \quad=$ Viscosidade

$\mu_{g} \quad=\quad$ Viscosidade do gás

$\mu_{g w} \quad=\quad$ Viscosidade do gás, avaliada a $\mathrm{P}_{\mathrm{wf}}$

$\bar{\mu}_{g} \quad=\quad$ Viscosidade média do gás, avaliada a $\overline{\mathrm{P}}$

$\rho=$ Massa específica

$\rho_{a r} \quad=$ Massa específica do ar

$\rho_{g} \quad=\quad$ Massa específica do gás

$v=\quad=$ Velocidade aparente

$\tau_{w} \quad=\quad$ Esforço cisalhante

$\Psi_{D} \quad=$ Pseudo pressão do gás real, para um $t_{D}$ 